\title{
Development and characterization of a superresolution ultrasonic transducer
}

\author{
José P. Leão-Neto, Everton B. de Lima, João H. Uliana, Theo Z. Pavan, Glauber T. Silva, and José H. Lopes
}

\begin{abstract}
Highly sensitive ultrasound probes are needed to expand the capabilities of biomedical ultrasound and industrial non-destructive testing (NDT). Pursuing better imaging quality, while keeping fabrication costs low, is an important trend in the current development of ultrasound imaging systems. In this paper, we report the development and characterization of an ultrasonic transducer that (super)focuses ultrasonic waves beyond the so-called diffraction limit, i.e., the beamwaist is roughly narrower than one wavelength. The transducer comprises an additive manufactured case with a circular flat piezoelectric actuator fixed at the bottom and a core-shell lens (with a stainless steel core and a polymer shell) placed at the probe's conical tip. The core-shell lens is responsible to superfocusing effect of ultrasonic waves. Operating at approximately $3 \mathrm{MHz}$, the transverse and axial resolution for C- and B-scan images are, respectively, $0.65 \lambda$ and $3 \lambda / 2$, with the wavelength being $\lambda=0.5 \mathrm{~mm}$. Whereas the system depth-of-field is $6.3 \lambda$. To demonstrate the transducer capability to resolve subwavelength structures, we successfully obtain images of a copper wire forming a Y-intersection, whose branches a diameter similar to human hair $(0.15 \mathrm{~mm} \approx 0.3 \lambda)$. Our results represent a solid step toward the development of ultrasonic superresolution transducer applied for biomedical imaging and shallow NDT of materials.
\end{abstract}

Index Terms-Subwavelength ultrasonic focusing, Diffraction limit, Superresolution imaging.

\section{INTRODUCTION}

$\mathbf{F}$ OCUSED ultrasonic fields are the cornerstone of ultrasound biomedical imaging [1]-[3], non-destructive testing of materials [4], and acoustic microscopy. The main role of a focused beam is to assess local fluctuations of mechanical properties, such as compressibility and density, of biological tissue and material failures in a region of interest (ROI). A key feature of ultrasonic imaging is the system resolution which corresponds to the ability to resolve discrete elements. The imaging resolution depends on many factors and properties of the system and scanned patient or object, which includes transducer geometry, frequency, and bandwidth, as well as post-processing electronics, and mechanical properties of the inquired medium. From the wave propagation point of view, the system resolution can be improved by tightly focusing the ultrasonic incident beam.

Conventional ultrasound focusing schemes are limited by diffraction. For example, the beam produced by a spherically focused transducer is limited to a circular spot, also known as

José P. Leão-Neto, and José H. Lopes are with Acoustics and Application Group, Federal University of Alagoas, Arapiraca, AL 57309-005, Brazil

Everton B. de Lima, and Glauber T. Silva are with Physical Acoustics Group, Institute of Physics, Federal University of Alagoas, Maceió, AL 57072970, Brazil

João H. Uliana, and Theo z. Pavan are with Department of Physics, FFCLRP, University of São Paulo, Ribeirão Preto 14040-901, SP, Brazil the Airy disk, of diameter [5] $d=1.22 F \lambda$, with $F$ and $\lambda$ being the transducer f-number (i.e., the focal distance to aperture ratio) and wavelength. Usually the f-number is larger than one, $F>1$. As a result of the diffraction limit just presented, two neighboring points can only be resolved by the spherically focused transducer if their inter-distance exceeds $1.22 \lambda F$. The easiest route to improve the ultrasonic resolution is to increase frequency. This follows at the expense of enhancing ultrasound attenuation as it varies with frequency squared in liquids, for instance.

Several techniques have aroused to deceive the diffraction limit including harmonic imaging [6], lens based on metamaterial [7]-[10] and phononic crystals [11]-[13], nonlinear ultrasound mixing [14]-[17], and time-reversal mirror [18][19]. Methods based on nonlinear properties of ultrasonic wave propagation such as second-harmonic generation and wave mixing have been successfully applied to biomedical imaging. The other subwavelength focusing methods are still finding some difficult to be established in the ultrasonic imaging realm. In part, because of the need for an elaborate hardware or challenges in metamaterial fabrication to meet beam focusing in the megahertz-frequency range.

Recently, a simple subwavelength focusing approach was proposed using a ball-shaped lens made of plastic (rexolite) with several wavelengths of diameter [20]. The apparatus yields a transverse resolution of about $\lambda / 2$. Also, a core-shell lens, with the outer layer made of a thermoplastic polymer and filled with ethanol, superfocused an ultrasound beam in water to a $0.85 \lambda$ spot [21]. Others arrangements using lenses with different geometries such as cylindrical, cuboid and Fresnel shapes yield a resolution even smaller than $\lambda / 2$ [22]-[31]. Ultrasonic images have successfully generated with the lens-based superresolution system [32]-[33]. Nonetheless, these experimental apparatuses are assembled by manually aligning a piezoelectric actuator and lens within a water tank. The consistent advancement toward a superresolution imaging system requires the development of a more robust superfocusing system comprising the actuator and lens in a single probe.

In this work, we fabricate and characterize a superresolution ultrasonic transducer comprising a piezoelectric actuator glued to $3 \mathrm{D}$ printed cylindrical case that works as a wave guide with a cone-trunk tip, where a core-shell lens is glued at the case tip. The transducer features were chosen out of a set of finite element simulations varying its geometric parameters. The transducer case is filled out with a coupling fluid, distilled water. It operates in both continuous $(\mathrm{CW})$ or pulsed mode, with operational frequency around $3 \mathrm{MHz}$. A set of careful 


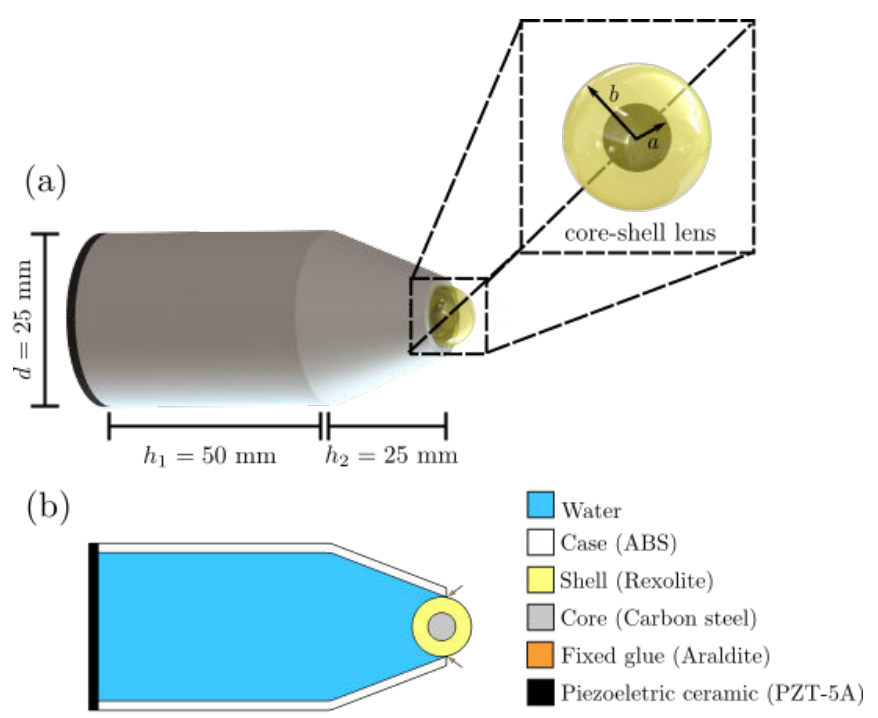

Fig. 1. (a) Schematic representation of the transducer with case height $h_{1}$, tip height $h_{2}$, and homemade narrow band piezoelectric actuator with diameter $d$ that is glued at the transducer's bottom. The inner and outer radii of the core-shell lens are denoted by $a$ and $b$, respectively. (b) The axial view of the transducer.

simulations and experiments are conducted to determine the system point spread function. The transverse resolution and depth-of-field obtained are, respectively, $0.65 \lambda$ and $6.3 \lambda$, with $\lambda \approx 0.5 \mathrm{~mm}$ at $3 \mathrm{MHz}$. The system focal distance is $16 \lambda$ away from the lens. To demonstrate the capabilities of the proposed system in both C- and B-scan modes, we image a copper wire forming a Y-intersection, where each branch as a $0.3 \lambda$ diameter. The subwavelength details of the splitting wire are well resolved in the obtained images. With superresolution capabilities in a relatively shallow region (i.e., a region close to the interface between the lens and scanned object), the proposed transducer may find applications in biomedical imaging and NDT of materials.

\section{MethodS AND MATERIALS}

\section{A. Finite element numerical model}

The choice of the transducer geometric parameters was guided by the results of obtained via finite element simulations, performed in the software platform COMSOL Multiphysics (Comsol AB, Sweden). The simulations were performed using the Pressure Acoustics and Solid Mechanics modules, which describe the wave propagation in water and elastic solid parts (case and core-shell lens), respectively. A harmonic excitation of $3 \mathrm{MHz}$ at the piezoactuator-inner fluid interface was adopted as it requires less memory usage of the computational system. We considered a mesh density of 15 points per wavelength, which corresponds to an error of $1.76 \%$ in the mesh convergence analysis. Finally, to avoid wave reflections due to the domain finite size, we set a perfect matched layer (PML) at the outer region of the external fluid.

\section{B. Superresolution ultrasonic transducer}

The transducer consists of three parts: a 3D printed case, a core-shell lens and a lead-zirconate-titanate-based piezoelec-

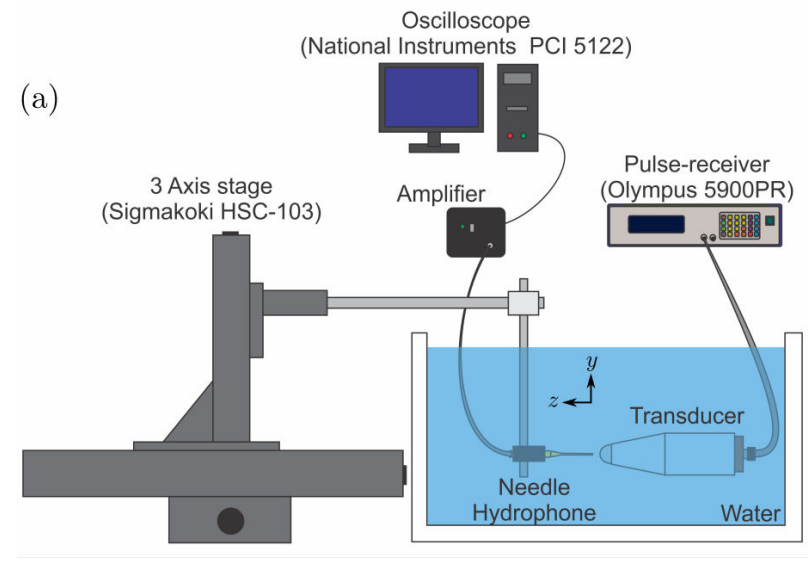

(b)

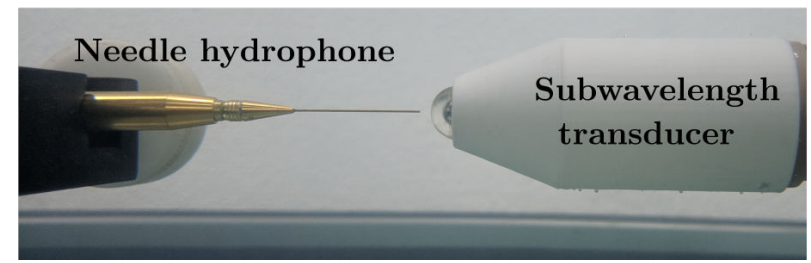

Fig. 2. (a) The experimental setup used to measure the pressure field generated by the superresolution transducer in a water tank. The radio-frequency (RF) ultrasound signal is generated by a pulse-receiver. The 3-axis stage system moves the needle hydrophone which acquires the RF ultrasound signal. The $\mathrm{RF}$ signal is then amplified, digitized, and stored in a computer. (b) Photograph of the fabricated device with the needle hydrophone.

tric (PZT-5A) actuator. The 3D printed case is a hollow cylinder made of acrylonitrile butadiene styrene (ABS) polymer of a $2 \mathrm{~mm}$ thickness. A carbon-steel core and rexolite shell lens with inner and outer radius of $a=3 \mathrm{~mm}$ and $b=6.4 \mathrm{~mm}$ is glued with araldite (Huntsman, Corp., USA) at the probe's conical tip of the case-see Fig. 1(a). The piezoelectric actuator, with $25 \mathrm{~mm}$ diameter, is glued at the open bottom of the case sealing the superresolution ultrasonic transducer. A backing layer was added to improve the actuator bandwidth. A mixture of $75 \%$ epoxy (AW106/HV853, Huntsman, Corp., USA) and tungsten powder was used to this end. A $\lambda / 4$-matching layer of epoxy (GY279/HY956, Huntsman, Corp., USA) was placed on top of piezoelectric actuator. The first measured resonance frequency of the piezo-actuator is nearly $3 \mathrm{MHz}$. The distance from the actuator the core-shell lens center is $75 \mathrm{~mm}$. The transducer cylindrical case is filled with a coupling fluid (distilled water). Fig. 1(b) shows the cross-section view along the axial of the transducer. Also, the physical parameters of the materials used in the transducer fabrication are presented in Table I. The transducer geometric parameters are chosen among the best results obtained from parametric numerical simulations of the system, by varying the height and tip conic angle. To this end, we opt to fabricate a device that is easy to both manual and robotic handling.

\section{Experimental setup and control electronics}

Figure 2(a) shows the experimental setup used for studying the subwavelength focused beam. In pulse-echo mode, the 
TABLE I

PHYSICAL PARAMETERS OF THE SUBWAVELENGTH TRANSDUCER

\begin{tabular}{lr}
\hline \hline Description & Value \\
\hline Piezoelectric actuator: PZT-5A & \\
Central frequency & $3 \mathrm{MHz}$ \\
Thickness & $1 \mathrm{~mm}$ \\
Density & $7750 \mathrm{~kg} \mathrm{~m}^{-3}$ \\
Longitudinal velocity & $4350 \mathrm{~m} \mathrm{~s}$ \\
Acoustical impedance & $33.7 \mathrm{MPa}$ \\
& \\
Backing layer: Tungsten + Epoxy (AW106/HV853) & $10 \mathrm{~mm}$ \\
Thickness & $1210 \mathrm{~kg} \mathrm{~m}^{-3}$ \\
Density & $2600 \mathrm{~m} \mathrm{~s}$ \\
Longitudinal velocity & $31.5 \mathrm{MPa}^{-}$ \\
Acoustical impedance & \\
Front layer: Epoxy (GY279/HY956) & $0.125 \mathrm{~mm}$ \\
Thickness & $1100 \mathrm{~kg} \mathrm{~m}^{-3}$ \\
Density & $2550 \mathrm{~m} \mathrm{~s}$ \\
Longitudinal velocity & $9 \mathrm{MPa}$ \\
Acoustical impedance & \\
\hline
\end{tabular}

transducer is driven by a pulse-receiver (5900PR, Olympus, USA) that produces pulses of $100 \mathrm{~V}$ amplitude at $100 \mathrm{~Hz}$ repetition rate, with $50 \Omega$ of electrical damping. All experiments take place in a water-tank of dimensions $100 \mathrm{~cm}(\mathrm{~L}) \times$ $100 \mathrm{~cm}(\mathrm{~W}) \times 150 \mathrm{~cm}(\mathrm{H})$. The generated ultrasonic pressure is measured with a calibrated needle hydrophone (HP series, Precision Acoustics, Dorchester, UK) with a $0.2 \mathrm{~mm}$ diameter. The hydrophone is attached to a computer controlled 3D positioning system (HSC-103, Sigmakoki, Co.,Ltd., Japan). The acquired RF signal is digitized by an A/D converter (PCI5122, NI, Inc. USA) and post-processed using MATLAB software (Mathworks, Inc., USA). To map the ultrasonic field, the hydrophone was raster-scanned in the system focal plane $(10 \mathrm{~mm} \times 10 \mathrm{~mm})$ in discrete steps of $0.05 \mathrm{~mm}$. Fig. 2(b) shows a photograph of the positioned needle hydrophone and the fabricated transducer immersed in the water tank.

\section{Image spatial resolution}

We now discuss that the superresolution transducer can be modeled as a linear system. The ultrasonic waves used to form images propagate in a homogeneous fluid of density $\rho_{0}$ and adiabatic speed of sound $c_{0}$. The focused beam can be locally approximated to a traveling plane wave; as a result, the corresponding fluid velocity is $v_{0}=p_{0} / \rho_{0} c_{0}$. For the sake of the argument, we have to consider $p_{0}$ as the peakpressure at the focus. All other pressures produced in the beamforming process, including those inside the transducer case, are assumed to be smaller than $p_{0}$. As long as the ratio of fluid velocity to the speed of sound is small, $v_{0} / c_{0} \ll 1$, we can safely consider the superresolution ultrasonic transducer as a linear system linear. We measured the peak pressure at the focus as $p_{0}=0.73 \mathrm{MPa}$, which gives the ratio $v_{0} / c_{0}=$ $\mathrm{O}\left(10^{-4}\right)$. Therefore, we conclude the waves produced by the transducer can be modeled with the linear acoustics theory.

By neglecting nonlinear effects in the image formation process, the transducer can be seen as a causal linear timeinvariant system. To describe the image formation process, we define a Cartesian coordinate system $O(x, y, z)$ at the center of the core-shell lens. Consider that an object or region to be imaged is represented by a function $f(\boldsymbol{r})$ of space only. The pressure in time-domain of the transmitted pulse by the transducer is denoted by $p_{\text {tr }}(\boldsymbol{r}, t)$. In pulse-echo mode, the RF ultrasound signal detected by the piezoelectric element from a small structure inside the imaging region at position $\boldsymbol{r}$ is given by [33]

$$
\begin{aligned}
s(\boldsymbol{r}, t) & =h(\boldsymbol{r}, t) \star f(\boldsymbol{r}), \\
h(\boldsymbol{r}, t) & =e_{\mathrm{t}-\mathrm{r}}(t) * p_{\mathrm{tr}}(\boldsymbol{r}, t) * p_{\mathrm{tr}}(\boldsymbol{r}, t),
\end{aligned}
$$

where $h(\boldsymbol{r}, t)$ is the system impulse response, $e_{\mathrm{t}-\mathrm{r}}(t)$ represents the lumped transmission-receive electromechanical response of the piezoelectric element (in units of $\mathrm{V} \mathrm{Pa}^{-2}$ ), which is also related to the type of the applied pulse. The asterisk and star symbols mean convolution in time and space, respectively. The impulse response $h(\boldsymbol{r}, t)$ also accounts for the diffraction effects in the image formation process and is, thus, recognized as the system point spread function (PSF). In short, this function represents the spreading of a point-like object due to diffraction. Expressing the system PSF in terms of the transmitted pressure $p_{\text {tr }}$ allows us to determine the system resolution by simply measuring this pressure.

It is more straightforward to analyze the spatial transducer resolution by taking the Fourier transform $\mathcal{F}$ of the impulse function $h(\boldsymbol{r}, t)$. We thus define the normalized PSF of the system as

$$
h_{\mathrm{PSF}}(\boldsymbol{r})=\left|\frac{\mathcal{F}[h(\boldsymbol{r}, t)]}{\mathcal{F}\left[h\left(\boldsymbol{r}_{\mathrm{f}}, t\right)\right]}\right|_{\omega=\omega_{0}}=\left|\frac{P_{\mathrm{tr}}\left(\boldsymbol{r}, \omega_{0}\right)}{P_{\mathrm{tr}}\left(\boldsymbol{r}_{\mathrm{f}}, \omega_{0}\right)}\right|^{2},
$$

where $\boldsymbol{r}_{\mathrm{f}}=\left(0,0, z_{\mathrm{f}}\right)$ points to the system focus, $\omega_{0}$ is the transducer operational frequency, and $P_{\text {tr }}$ is the frequency spectrum of the transmitted pressure.

The transmitted pressure is measured by a detector placed at $\boldsymbol{r}$. The corresponding electric signal is denoted by $s_{\mathrm{tr}}(\boldsymbol{r}, t)$, to which the analytic representation is obtained through the Hilbert transform $\mathcal{H}$,

$$
\tilde{s}_{\mathrm{tr}}(\boldsymbol{r}, t)=s_{\mathrm{tr}}(\boldsymbol{r}, t)+\mathrm{i} \mathcal{H}\left[s_{\mathrm{tr}}(\boldsymbol{r}, t)\right]=\mathrm{e}^{-\mathrm{i} \omega_{0} t} g(\boldsymbol{r}, t),
$$

with $g$ being envelope function of the transmitted pulse. The Fourier component of the transmitted pressure at $\omega=\omega_{0}$ reads

$$
P_{\mathrm{tr}}^{2}\left(\boldsymbol{r}, \omega_{0}\right)=\mathcal{F}[g(\boldsymbol{r}, t)]_{\omega=0}=E_{\mathrm{t}-\mathrm{r}}^{-1}\left(\omega_{0}\right) \int_{-\infty}^{\infty}\left|\tilde{s}_{\mathrm{tr}}(\boldsymbol{r}, t)\right| \mathrm{d} t,
$$

where $E_{\mathrm{t}-\mathrm{r}}$ is the Fourier transform of the lumped transmissionreceive electromechanical response of the piezoelectric element. After measuring the transmitted pressure, we can use this equation into Eq. (2) to determine the system PSF.

Finally, we can determined the system spatial resolution by noting, the transmitted pulse has circular symmetry around the $z$ axis. The transverse resolution $w$ and depth-of-field $d$ of the system are defined as the full width at half maximum (FWHM) of the system PSF, which can be determined from the relations

$$
h_{\mathrm{PSF}}\left(\frac{w}{2}, z_{\mathrm{f}}\right)=h_{\mathrm{PSF}}(0, d)=\frac{1}{2} .
$$




\section{E. $B$ - and $C$-scan imaging modes}

To form B- and C-scan images, the system acquires A-lines, which are represented by the envelope of the received RF signal $s_{\text {rec }}(t)$ by the transducer piezoelectric element [34]

$$
e_{\text {recv }}(t)=\left|s_{\text {recv }}(t)+\mathrm{i} \mathcal{H}\left[s_{\text {recv }}(t)\right]\right| .
$$

This function is logarithmically compressed to reduce the dynamic range of the detected RF signal. By raster-scanning an object centered at the focal plane, we can form a $3 \mathrm{D}$ image of the object with $L \times M \times N$ voxels.

The acquired echo signals at each scanning position (Ascan) are digitized with a sampling frequency of $f_{s}=20 \mathrm{MHz}$. The signal is then post-processed in MATLAB programming language (MathWorks, Inc., USA), to form the ultrasound images. The B-scan image of an object is generated by combining 60 A-scan lines with lateral steps of $0.05 \mathrm{~mm}$. The C-scan images of the system correspond to a region in the transducer focal plane, $z=z_{\mathrm{f}}$. The lateral resolution of both B- and C-scan images depends on the scanning step and transverse width of the transmitted ultrasound beam. The axial resolution of B-scan images is related the half duration of the transmitted RF pulse.

\section{RESULTS AND DISCUSSIONS}

\section{A. Finite element simulation results}

In Fig. 3, we show the influence of the transducer height $h$ on the ultrasonic focused beam. Panel (a) presents the schematic view of the transducer, and panel (b) and (c) show the axial and lateral beam intensity, respectively, for $h=10,23,50 \mathrm{~mm}$. We see that the depth-of-field (intensity at $-3 \mathrm{~dB}$ ) relative change is less than $10 \%$. In particular, the largest value $(d=3 \mathrm{~mm})$ occurs at $h=50 \mathrm{~mm}$. On the other hand, the beamwaist does not change with height.

In Fig. 4 we present the simulation results for the tip conic angles $\theta=65^{\circ}, 75^{\circ}$. The transverse beam profile does not visibly change with the tip angle. While the depth-of-field shows a relative variation of about $10 \%$.

It is worth mentioning that a subwavelength focused beam was achieved for all results presented here, with a beamwaist of about $0.3 \mathrm{~mm}$. Therefore, we chose to fabricate a transducer with $h=50 \mathrm{~mm}$ and $\theta=75^{\circ}$ also because it is can be both manually and robotically handle for future imaging purposes.

\section{B. Subwavelength point spread function}

In Fig. 5, we show the RF ultrasound signals (given in volts) measured by the needle hydrophone along the transducer axial direction at $z=6.5,8.0,11.4,13.4,16.4 \mathrm{~mm}$, with the corresponding labels as $1-5$. We recognize two well-resolved pulses in each detected RF signal. The first pulse arriving at an earlier time in the hydrophone is a spurious signal that travels along the transducer ABS case. This signal accompanies the superfocused (main) pulse and its amplitude decays with the inverse of distance to the transducer. At the focal region, the spurious pulse is $-8 \mathrm{~dB}$ of the main pulse, as seen in Fig. 5, signal 2. With the wave propagation speed in ABS
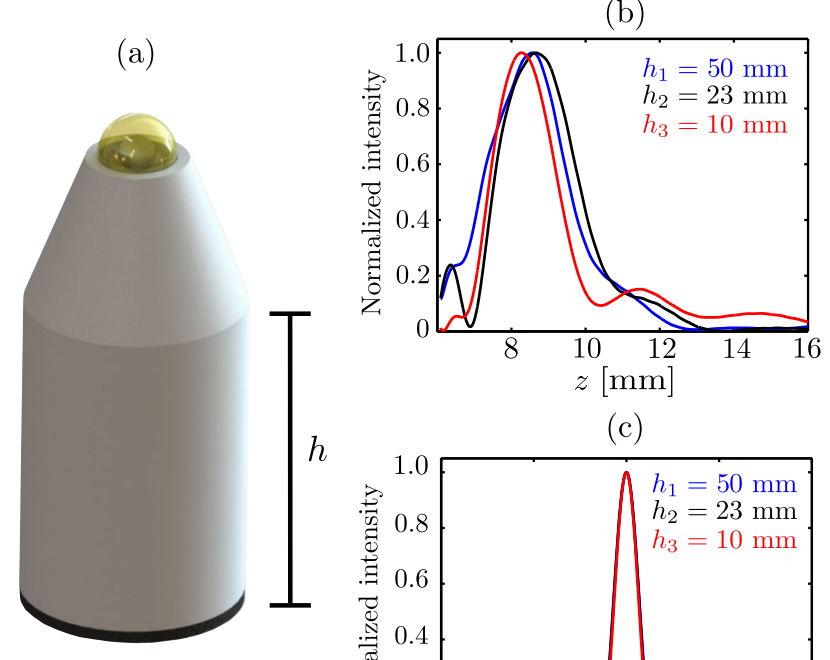

(c)

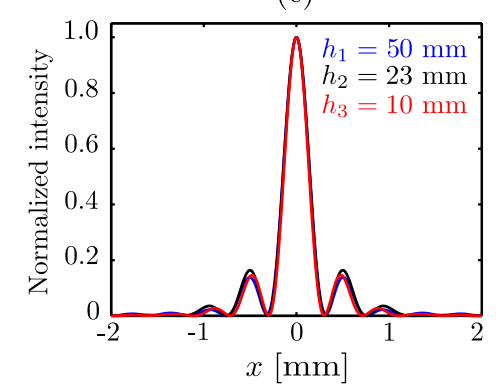

Fig. 3. Numerical result of the beam profile with different transducer height. (a) Schematic representation of the transducer height. (b) Axial and (c) transverse beam intensity.

(b)
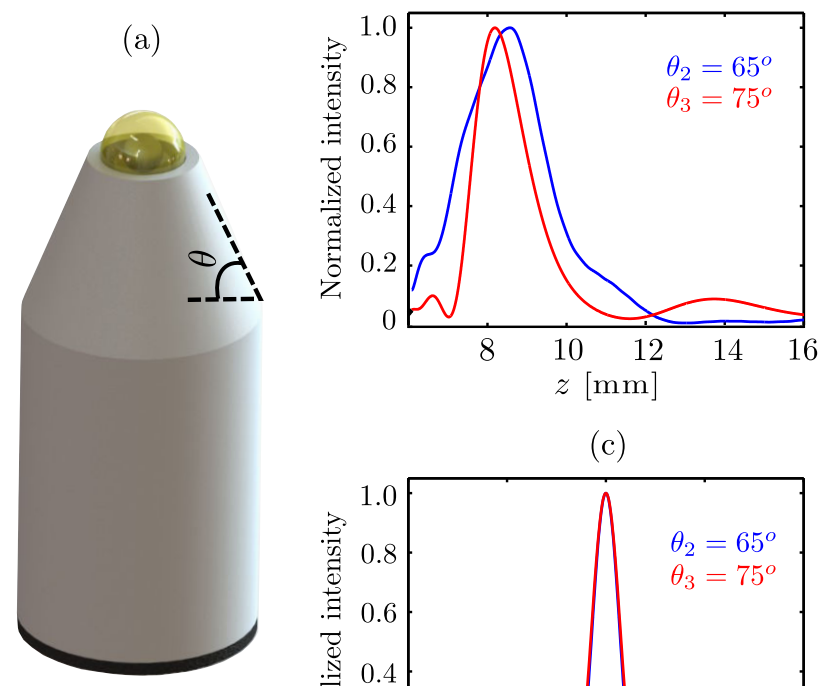

(c)

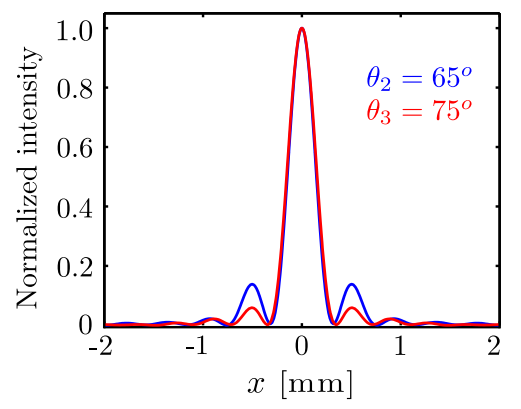

Fig. 4. Numerical result of the beam profile with two different tip conic angle. (a) Schematic representation of the device conic angle. (b) Axial and (c) transverse beam intensity. 

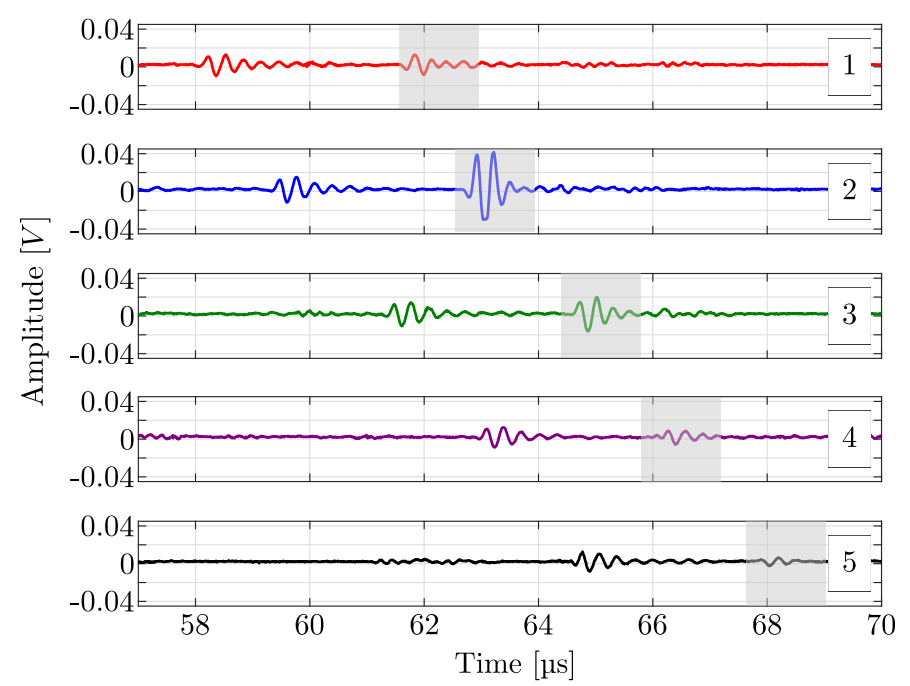

Fig. 5. The transmitted RF signal by the transducer measured with a needle hydrophone. The signals are detected at $z=6.5,8.0,11.4,13.4,16.4 \mathrm{~mm}$ along the axial direction, with the corresponding labels as $1-5$. The first pulse that arrives in the hydrophone is due the wave propagation in the transducer case. The second pulse gives rise to the subwavelength focused beam. The gray boxes represent moving gate of $1.5 \mu$ s duration.

is $2040 \mathrm{~m} \mathrm{~s}^{-1}$, which is larger than that in water $1500 \mathrm{~m} \mathrm{~s}^{-1}$, spurious pulses arrive faster at the hydrophone. The second pulse, framed in a gray box and referred to as the main pulse, gives rise to the subwavelength focused beam by the core-shell lens. The time difference between the spurious and main pulses is $3.6 \mu \mathrm{s}$, which remains constant in all RF signals presented in Fig. 5. In signals 4 and 5, the spurious pulse becomes more prominent. It is then clear we need to eliminate the influence of the spurious pulse.

To measure the system PSF, we eliminate the spurious signal using a moving gate (gray box) around the main pulse with a duration of $1.5 \mu \mathrm{s}$. This procedure was performed only to measure the transducer PSF. We emphasize the spurious pulse may cause image artifacts when applying the superfocusing transducer to image complex media such as biological tissues or layered materials. This limitation might be overcome by improving the coupling geometry between the lens and case, and also by using a solid material as the transducer bulk. However, we used a simpler approach based on gating the detected RF signal, which reduces the effects of spurious pulses.

In Fig. 6, we present the system PSF measured numerically and experimentally by a needle hydrophone along with finite element results. The operational frequency of the transducer is approximately $3 \mathrm{MHz}$. The PSF is computed through Eq. (2), mean value of the transmitted (gated) signal is calculated yielding the Fourier component of the transmitted pressure according to Eq. (4). Panel (a) illustrates the experimental 2D PSF in the axial plane. Panels (b) and (c) show the comparation of the numerical and experimental PSF along the axial and transverse directions, respectively, as depicted by white dashed lines in panel (a). We see that the depth-of field is $d=3.15 \mathrm{~mm}=6.3 \lambda$, while the transverse resolution is $w=0.33 \mathrm{~mm}=0.65 \lambda$. This demonstrates that the ultrasonic

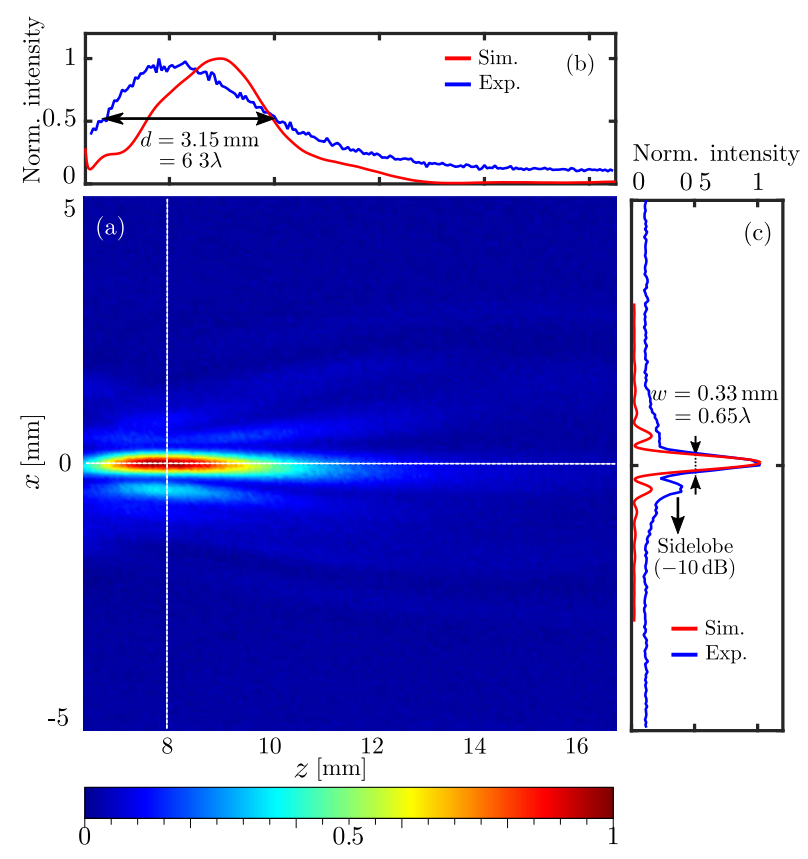

Fig. 6. (a) The system PSF obtained experimentally by a needle hydrophone in the axial plane ( $x z$ plane). The horizontal and vertical white dotted lines depict the axial and transverse direction crossing at the system focus. (b) The numerical (red solid line) and experimental (blue solid line) of the PSF along the axial direction. The depth-of-field is about $d=6.3 \lambda$. (c) The numerical (red solid line) and experimental (blue solid line) the PSF in the transverse direction at the focal plane is $w=0.65 \lambda$.

beam is indeed focused beyond the diffraction limit, $w<\lambda$. We also note the presence of a sidelobe of about $-10 \mathrm{~dB}$. The asymmetric sidelobe may cause a target to appear as a central spot followed by an irregularly distributed ring. It thus may produce asymmetric shapes on an image. The observed asymmetry in the system PSF might be caused by a misalignment of the transducer parts in the assembly process.

The alignment error between the lens and 3D-printed case can be minimized using a micrometer-resolution positioning system to assist the device assembly.

\section{Superresolution images}

To show that the transducer is able to resolve subwavelength structures of an object, we image a copper wire forming a Y-junction. The wire, with a diameter of $0.15 \mathrm{~mm}=0.3 \lambda$, is stretched in the system focal plane. The transducer rasterscans the wire acquiring $200 \times 60$ A-lines with linear steps of $0.05 \mathrm{~mm}$. The corresponding RF ultrasound signal of each A-line has duration of $50 \mu \mathrm{s}$. Note that to avoid reverberations in the internal structure of the lens to the PZT transducer, we perform a background subtraction from the obtained RF data. The background data is acquired by scanning the same region without the presence of the copper wire. To form the ultrasonic images, the RF echo is gated in a window of $2.5 \mu \mathrm{s}$, which reduces the effects of spurious pulses from the transducer case. The initial time of the gated signal is computed from the timeof-flight $(\approx 100 \mu \mathrm{s})$ of a pulse-echo response. As the system has fixed focus, we can keep the gate position regardless of the imaged object. We notice from experimental measurement 


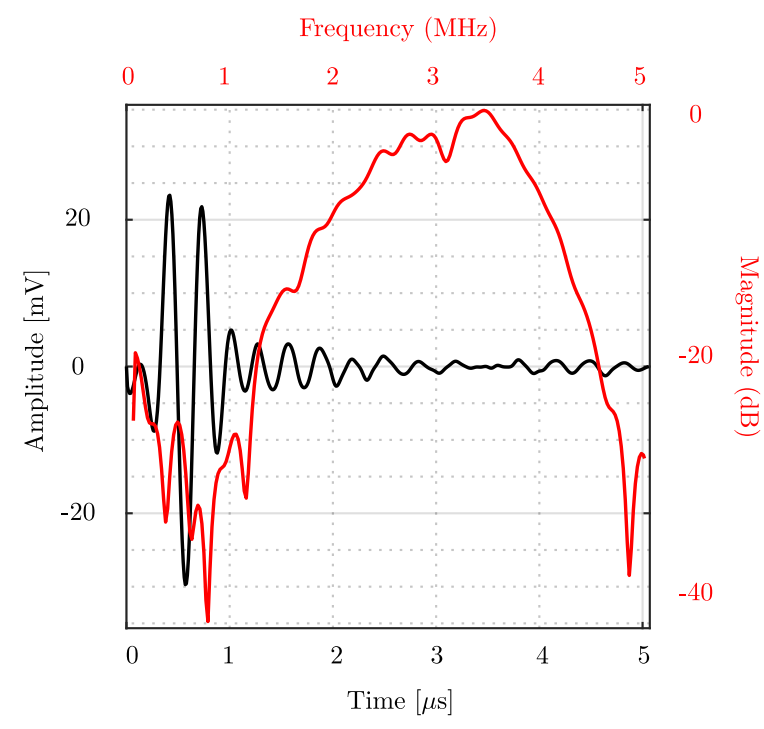

Fig. 7. Pulse-echo response of the transducer for the copper wire (black line) and corresponding spectrum (red line).

that the time duration of the gated signal $2.5 \mu$ s safely encloses the echo response of our system.

The axial resolution of the B-scan mode is determined by taking half of pulse duration $(\Delta t)$. According to Fig. 7 , the received $\mathrm{RF}$ pulse has three cycles above $-12 \mathrm{~dB}$, with a time interval of $\Delta t=1 \mu \mathrm{s}$. So the resolution is $c_{0} \Delta t / 2=3 \lambda / 2=$ $c_{0} / \Delta f=0.75 \mathrm{~mm}$, where $\Delta f=1.8 \mathrm{MHz}$ is the transducer bandwidth at $-6 \mathrm{~dB}$.

Figure 8 shows a photograph of the phantom and transducer with the copper wire placed at the system focal plane. In Fig. 9, panel (a) and (b) show the C- and B-scan image of the copper wire, respectively. In panel (c), we present the transducer in position to scan the copper wire. The C-scan image in panel (a) shows the Y-junction with excellent detail. The B-scan image, taken at the white dashed line in panel (a), shows two branches of the wire. The image of the left wire branch has a width and height of of $0.33 \mathrm{~mm} \approx 0.65 \lambda$ and $0.72 \mathrm{~mm} \approx$ $3 \lambda / 2$, respectively. This is compatible with the lateral and axial resolutions of the B-scan mode of the system. On the other hand, the image of the right wire branch appears slightly larger than the left branch due to irregular shape of the wire. Note also that the dynamic range of the system is $29.8 \mathrm{~dB}$.

In Fig. 10, the 3D superresolution image of the copper wire formed by rendering the $60 \times 20$ A-lines acquired by the system. We clearly see the subwavelength details in the bifurcation region between $4 \mathrm{~mm}$ to $6 \mathrm{~mm}$ along the wire.

\section{CONCLUSiON}

In this research, a superresolution ultrasonic transducer has been designed, fabricated, characterised and tested. The device assembly comprises a $3 \mathrm{MHz}$ flat transducer glued in an 3D printed cylindrical case with a cone-trunk tip, where a coreshell lens is glued. To characterize the beam produced by the transducer a gate of $1.5 \mu \mathrm{s}$ was done around the main signal. This process was realized to eliminate the spurious signal from the ABS case. The subwavelength focusing beam generated

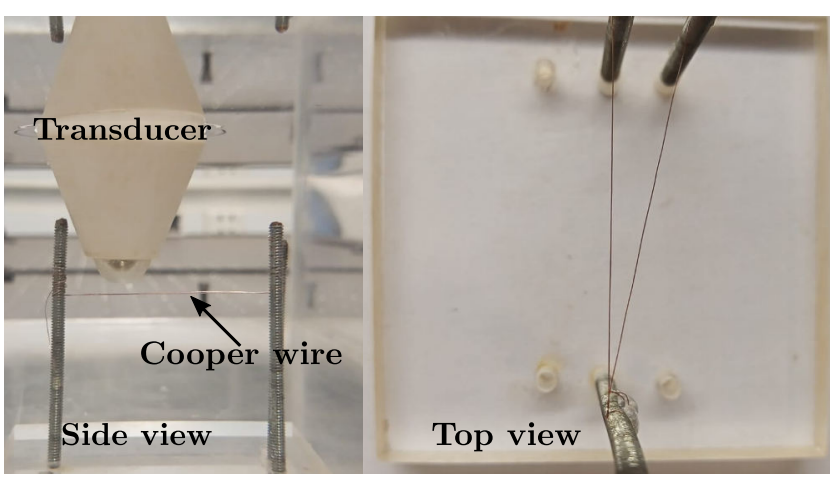

Fig. 8. A photograph of the phantom and transducer with the copper wire placed at the system focal plane.

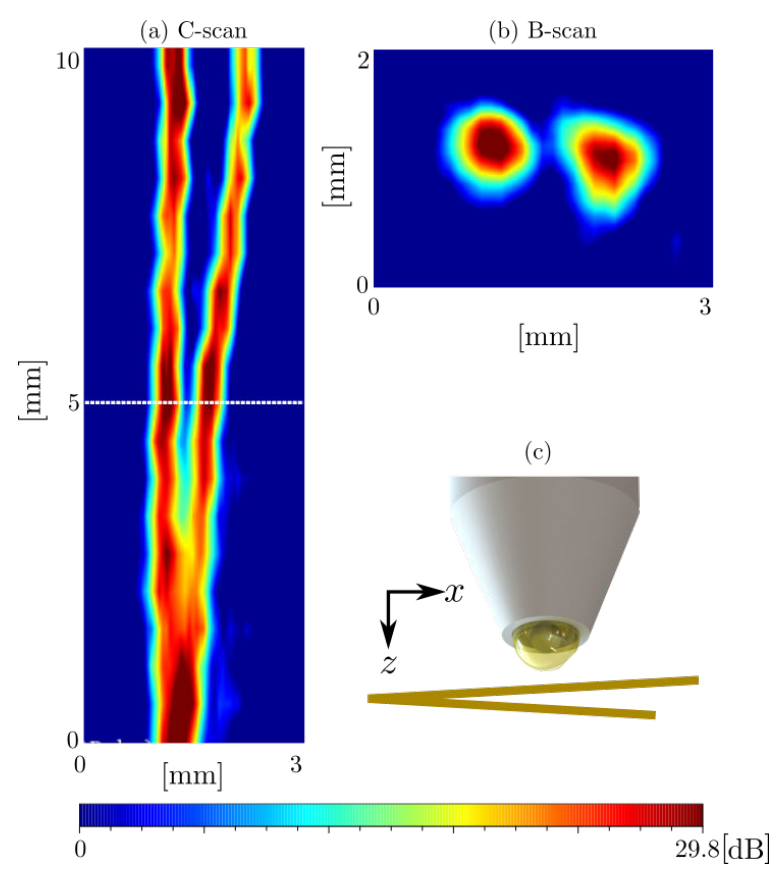

Fig. 9. Superresolution image of the copper wire immersed in water. (a) $\mathrm{C}$-scan image. (b) B-scan image related to the transverse plane depicted by the white dashed white line. The dark blue and dark red colors correspond to minimum and maximum pixel values. The dynamic range of the image is $29.8 \mathrm{~dB}$. (c) A sketch of the copper wire placed at the system focal plane.

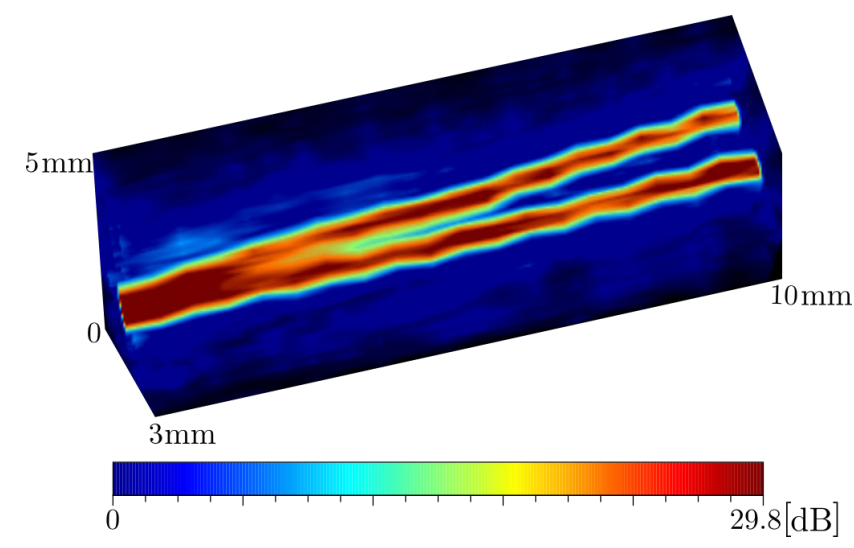

Fig. 10. Superresolution 3D image of the copper wire formed with $60 \times 20$ A-scan lines. 
by the transducer was characterized computing its PSF (w $=0.65 \lambda$ ) and depth-of-focus $d=6.3 \lambda$. These values implies that our system working beyond the diffraction limit. Furthermore, to demonstrate the capabilities of the proposed device, we experimentally imaging a cooper wire with $0.15 \mathrm{~mm}$ of diameter immersed in water. B- and C- scan images were presented and the subwavelength details of the splitting wire are clearly observed.

The transducer presented in this work may find fruitful applications in ophthalmology and dermatology imaging diagnostic which requires shallow image scanning, and detecting near-surface flaws in materials. Also, this method might be suitable for enhancing acoustic microscopy resolution while keeping the same operational frequency and electronic hardware.

\section{ACKNOWLEDGMENT}

This work was partially supported by the Brazilian agency Conselho Nacional de Desenvolvimento Científico e Tecnológico-CNPq (Grants No. 311107/2020-6, No. 309375/2018-5 and No.437757/2018-8).

\section{REFERENCES}

[1] G. Lockwood, D. Turnball, D. Christopher, and F. Foster, "Beyond $30 \mathrm{mhz}$ applications of high-frequency ultrasound imaging," Eng. Med. Biol. Mag., IEEE, vol. 15., no. 6, pp. 60-71, 1996.

[2] C. Passmann and H. Ermert, "A 100-mhz ultrasound imaging system for dermatologic and ophthalmologic diagnostics," IEEE Trans. Ultrason., Ferroelect., Freq. Contr., vol. 43, no. 4, pp. 545-552, 1996.

[3] A. Webb and G. C. Kagadis, "Introduction to biomedical imaging," Med. Phys, vol. 30, pp. 2267-2267, 2003.

[4] J. Blitz and Simpson, Ultrasonic Methods of Non-destructive Testing, N. Springer, Ed. Springer, 1995, vol. 2.

[5] G. S. Kino, Acoustic Waves: Devices, Imaging and Analog Signal Processing. Englewood Cliffs, NJ.: Prentice-Hall, 1987.

[6] F. Tranquart, N. Grenier, V. Eder, and L. Pourcelot, "Clinical use of ultrasound tissue harmonic imaging," Ultrasound in Medicine \& Biology, vol. 25, pp. 889-894, 1999.

[7] S. Zhang, L. Yin, and N. Fang, "Focusing Ultrasound with an Acoustic Metamaterial Network," Phys. Rev. Lett., vol. 102, p. 194301, 2009.

[8] J. Li, L. Fok, X. Yin, G. Bartal, and X. Zhang, "Experimental demonstration of an acoustic magnifying hyperlens," Nat. Materials, vol. 8, pp. 931-934, 2009.

[9] J. Zhu, J. Christensen, J. Jung, L. Martin-Moreno, X. Yin, L. Fok, $X$. Zhang, and F. J. Garcia-Vidal, "A holey-structured metamaterial for acoustic deep-subwavelength imaging," Nat. Comm., vol. 7, pp. 52-55, 2011.

[10] T. Liu, F. Chen, S. Liang, H. Gao, and J. Zhu, "Subwavelength sound focusing and imaging via gradient metasurface enabled spoof surface acoustic wave modulation," Physical Review Applied, vol. 11, p. 034061, 2019.

[11] S. Yang, J. H. Page, Z. Liu, M. L. Cowan, C. T. Chan, and P. Sheng, "Focusing of Sound in a 3D Phononic Crystal," Phys. Rev. Lett., vol. 93, p. 024301, 2004.

[12] A. Sukhovich, L. Jing, and J. H. Page, "Negative refraction and focusing of ultrasound in two-dimensional phononic crystals," Physical review B, vol. 77, p. 014301, 2008.

[13] J. F. Robillard, J. Bucay, P. A. Deymier, A. Shelke, K. Muralidharan, B. Merheb, J. O. Vasseur, A. Sukhovich, and J. H. Page, "Resolution limit of a phononic crystal superlens," Physical review B, vol. 83, p. 224301, 2011.

[14] M. Fatemi and J. F. Greenleaf, "Ultrasound-stimulated vibro-acoustic spectrography," Science, 1998.

[15] F. G. Mitri, G. T. Silva, J. F. Greenleaf, and M. Fatemi, "Simultaneous sum-frequency and vibro-acoustography imaging for nondestructive evaluation and testing applications," Journal of Applied Physics, vol. 102, no. 11, p. 114911, 2007.
[16] G. T. Silva and F. G. Mitri, "Difference-frequency generation in vibroacoustography," Phys. Med. Biol., vol. 56, pp. 5985-5993, 2011.

[17] A. L. Baggio, H. A. S. Kamimura, J. H. Lopes, A. A. O. Carneiro, and G. T. Silva, "Parametric array signal in confocal vibro-acoustography," Appl. Acoustics, vol. 126, pp. 143-148, 2017.

[18] M. Fink, D. Cassereau, A. Derode, C. Prada, P. Roux, M. Tanter, J.L. Thomas, and F. Wu, "Time-reversed acoustics," Rep. Prog. Phys., vol. 63, pp. 1933-1995, 2000.

[19] G. Lerosey, J. de Rosny, A. Tourin, and M. Fink, "Focusing beyond the diffraction limit with far-field time reversal," Scienc, vol. 315, pp. $1120-1122,2007$

[20] J. H. Lopes, M. A. B. Andrade, J. P. Leão-Neto, J. C. Adamowski, I. V. Minin, and G. T. Silva, "Focusing acoustic beams with a ball-shaped lens beyond the diffraction limit," Phys. Rev Applied, vol. 8, p. 024013 2017.

[21] S. Pérez-López, P. Candelas, J. M. Fuster, C. Rubio, O. V. Minin, and I. V. Minin, "Liquid-liquid core-shell configurable mesoscale spherical acoustic lenswith subwavelength focusing," Applied Physics Express, vol. 12, p. 087001, 2019.

[22] D. V. Canle, T. Kekkonen, J. Mäkinen, T. Puranen, H. J. N. A. Kuronen, S. Franssila, T. Kotiaho, A. Salmi, and E. Hæggström, "Practical realization of a sub lambda/2 acoustic jet," Scientific Reports, vol. 9, p. 5189,2019

[23] S. Castiñeira-Ibáñez, D. Tarrazó-Serrano, P. Candelas, O. Minin, C. Rubio, and I. Minin, " $3 \mathrm{~d}$ sound wave focusing by $2 \mathrm{~d}$ internal periodic structure of 3d externalcuboid shape," Results in Physics, vol. 15, p. $102582,2019$.

[24] C. Rubio, D. Tarrazó-Serrano, O. V. Minin, A. Uris, and I. V. Minin, "Acoustical hooks: A new subwavelength self-bending beam," Results in Physics, vol. 16, p. 102921, 2020.

[25] S. Castiñeira-Ibáñez, D. Tarrazó-Serrano, A. Uris, C. Rubio, O. V. Minin, and I. V. Minin, "Cylindrical 3d printed confgurableultrasonic lens for subwavelengthfocusing enhancement," Scientific Reports, vol. 10, p. $20279,2020$.

[26] D. Tarrazó-Serrano, C. Rubio, O. V. Minin, A. Urisa, and I. V. Minin, "Ultrasonic focusing with mesoscale polymer cuboid," Ultrasonics, vol. 106 , p. 106143,2020

[27] D. Tarrazó-Serrano, C. Rubio, O. V. Minin, P. Candelas, and I. V. Minin, "Manipulation of focal patterns in acoustic soret type zone plate lens by using referenceradius/phase effect," Ultrasonics, vol. 91, pp. 237-241, 2019.

[28] S. Pérez-López, J. M. Fuster, P. Candelas, D. Tarrazó-Serrano, S. Castiñeira-Ibáñez, and C. Rubio, "Bifocal ultrasound focusing using bi-fresnel zoneplate lenses," Sensors, vol. 20, p. 705, 2020.

[29] C. Rubio, D. Tarrazó-Serrano, O. V. Minin, A. Uris, and I. V. Minin, "Sound focusing of a wavelength-scale gas-filled flat lens," EPL (Europhysics Letters), vol. 123, p. 64002, 2018.

[30] S. Castiñeira-Ibañez, D. Tarrazó-Serrano, A. Uris, and C. Rubio, "Tunable acoustic hooks from janus cylinder," Results in Physics, vol. 24, p. 104134, 2021.

[31] C. Rubio, D. Tarrazó-Serrano, O. V. Minin, A. Uris, and I. V. Minin, "Wavelength-scale gas-filled cuboid acoustic lens with diffraction limitedfocusing," Results in Physics, vol. 12, pp. 1905-1908, 2019.

[32] J. P. Leão-Neto, G. S. Cardoso, A. S. Marques, M. A. B. Andrade, J. C. Adamowski, T. Z. Pavan, G. T. Silva, and J. H. Lopes, "Subwavelength focusing beam and superresolution ultrasonic imaging using a core-shell lens," Physical Review Applied, vol. 13, p. 014062, 2020.

[33] E. B. Lima, V. H. Santos, A. L. Baggio, J. H. Lopes, J. P. Leão-Neto, and G. T. Silva, "An image formation model for ultrasound superresolution using a polymer ball lens," Applied Acoustics, vol. 170, p. 107494, 2020.

[34] T. L. Szabo, Diagnostic Ultrasound Imaging: Inside Out, J. Bronzino, Ed. Elsevier Academic Press, 2004. 